\title{
An EEG-based Brain Computer Interface for Rehabilitation and Restoration of Hand Control following Stroke Using Ipsilateral Cortical Physiology
}

\author{
Sam Fok, Student Member, IEEE, Raphael Schwartz, Mark Wronkiewicz, Charles Holmes, Jessica \\ Zhang, Thane Somers, Student Member, IEEE, David Bundy, Eric Leuthardt
}

\begin{abstract}
The loss of motor control severely impedes activities of daily life. Brain computer interfaces (BCIs) offer new possibilities to treat nervous system injuries, but conventional BCIs use signals from primary motor cortex, the same sites most likely damaged in a stroke causing paralysis. Recent studies found distinct cortical physiology associated with contralesional limb movements in regions distinct from primary motor cortex. To capitalize on these findings, we designed and implemented a BCI that localizes and acquires these brain signals to drive a powered, hand orthotic which opens and closes a patient's hand.
\end{abstract}

\section{INTRODUCTION}

troke is the leading cause of disability in the United States, with over 700,000 individuals affected annually [1]. Therapies requiring an active role of the patient in controlling their impaired limb are most likely to induce reorganization of neural pathways and improve motor control but require intensive patient-practitioner interaction [2]. Conventional, post-stroke physical therapy techniques are only marginally effective at restoring limb control.

The field of brain-computer interfaces (BCIs) holds the promise of new, more effective motor therapies. BCIs are traditionally applied to cases in which central nervous signals are cut off from their destination by injury by recording electrical signals directly from the brain to circumvent injured pathways and control devices that actuate the target limb. BCIs can thus couple intent to move a limb and movement of an orthosis in stroke therapy. This provides positive feedback for the patient and increase motivation and independence in recovery when compared current techniques.

Brain signal acquisition techniques range in degree of invasiveness. More invasive techniques give better spatial and temporal resolution but face significant technical barriers, clinical risks, and regulatory hurdles in their

Manuscript submitted for review April 15, 2011. This work was supported in part by the National Collegiate Inventors and Innovators Alliance (NCIIA), the Washington University in St. Louis School of Engineering, and Emotiv Systems.

Sam Fok and Raphael Schwartz are with the Biomedical Engineering and Electrical Engineering Departments, Washington University in St. Louis, MO 63130 USA. (phone: 314-341-4030; e-mail: sbfok@wustl.edu).

Mark Wronkiewicz, Charles Holmes, Jessica Zhang, and Thane Somers are with the School of Engineering, Washington University in St. Louis, MO 63130 USA.

David Bundy is with the Biomedical Engineering Department, Washington University in St. Louis, MO 63110 USA. (email: dtbundy@wustl.edu).

Eric Leuthardt is with the Department of Neurosurgery, Washington University in St. Louis, MO 63110 USA. (email: leuthardte@wustl.edu). implementation. Yet, despite relatively low spatial and temporal resolution, researchers have been able to obtain control in 2 and 3 dimensions using EEG signals [2].

While traditional BCI devices have been applied to a variety of neuromuscular disorders such as amyotrophic lateral sclerosis, cerebral palsy, and spinal cord injury, application to stroke victims has been problematic because stroke damaged cortex will not produce the signals used conventionally for BCIs, namely signals from the primary motor cortex contralateral, or opposite, to the affected limb. However, recent neurophysiological study has found distinct cortical physiology associated with ipsilateral, contralesional hand and limb movements in regions distinct and separable from the primary motor cortex [3, 4].

The design presented here is to demonstrate the feasibility of synthesizing recent neurophysiology and consumer available electronics to develop more effective stroke treatment. Implementation of this project constitutes a fundamentally new approach to restoring function in hemispheric stroke survivors by enabling the unaffected side of the brain to control their stroke impaired hand.

\section{TECHNICAL DESCRIPTION}

Overview

The system overview is displayed in Figure 1



Figure 1- Signal pathway of system

Synchronous firings of neurons over large areas of the brain are recorded on the scalp and sent to a laptop for processing. After a short, one-time calibration period, a frequency and electrode best corresponding to attempted hand movement is selected. This signal is processed to generate a control signal, which is then sent to a linear actuator controlled orthosis on a patient's hand, so that brainwaves can control the opening and closing of the hand. 
Visual feedback from BCI2000 and tactile feedback from the orthosis are provided to the user.

\section{A. Signal Acquisition}

EEG signals were acquired from the subject's scalp using a commercially available, dry-electrode Emotiv EPOC (Emotiv; Australia) headset, which comes preconfigured with 14 electrodes located over 10-20 international system positions AF3, F7, F3, FC5, T7, P7, O1, $\mathrm{O} 2, \mathrm{P} 8, \mathrm{~T} 8, \mathrm{FC} 6, \mathrm{~F} 4, \mathrm{~F} 8$, and AF4 using 2 reference electrodes (Figure 2). This arrangement has good coverage of the pre-motor and frontal regions of brain, which are the only areas of interest for this study. Clinical EEG caps would give better coverage of motor cortex, but cost, long setup time, and lack of portability prevent this approach for becoming a practical commercial solution. The headset aligns, bandpass filters, and digitizes the signal at $128 \mathrm{~Hz}$ before transmitting wirelessly to a laptop.

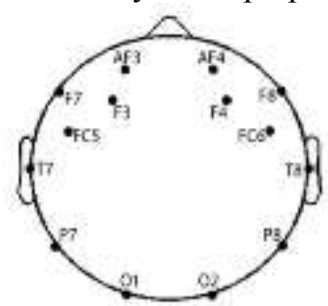

Figure 2 - Spatial location of electrodes of Emotiv EPOC headset relative to head

\section{B. Screening Procedure, Signal Processing and Control}

On a laptop, signal processing was carried out in the BCI2000 framework [5]. An initial screening in which signals are recorded while the subject makes hand movements is used to determine the EEG features to be used to distinguish movement from rest. During this screening procedure the user alternates between periods of attempted hand movement and periods of rest. After several trials, we identify the specific electrode channels and frequency bins which consistently changed in power between hand movement and rest conditions

A bipolar reference [6] at $6-9 \mathrm{~cm}$ distance is used to attenuate noise and artifacts on the scalp common to pairwise electrodes and to detecting signals from a region consistent in size with the diffuse spatial characteristics of EEG signals. This reference technique also makes the system resilient to variation in electrode placement. Five electrodes are available above the frontal lobe and premotor area of the contralesional hemisphere, the location of our signal of interest. Using these 5 electrodes, all combinations of spatial reference choices can be evaluated. The coefficient of determination $R^{2}$ is used as a measure of spatial reference performance, where $R^{2}$ indicates the proportion of variability in the signal attributable to the user's hand movement. The bipolar derivation is defined,

$$
B_{i j}=x_{i}-x_{j}
$$

Where $i=1 \ldots 5, j=1 \ldots 5$ refer to the electrode locations of interest and $B_{i j}$ is bipolar derivation of $i$ referenced to $j$. We evaluate each combination and select for the highest $R^{2}$,

$$
B_{\text {select }}=\arg \max \left(R^{2}\left\{B_{i j}\right\}\right)
$$

Once the bipolar reference has been selected, the frequency bin of width $2 \mathrm{~Hz}$ with the highest $R^{2}$ is then selected as the control spectrum. The bipolar spatial filtered recording is converted to actuator control signal through a continuously adapting least mean squares (LMS) regression algorithm.

\section{Least Mean Squares Adaptive Filter}

The LMS algorithm linearly scales the selected bipolar channel with gain $\mathrm{G}_{\mathrm{k}}$. The algorithm adjusts the gain after each trial to values which would have yielded better results in the previous trial. This is expressed as

$$
G_{k}=(1-\mu) G_{k-1}+\mu \frac{(\Delta L)_{i}}{S_{\text {mean }}}
$$

where $\mathrm{G}_{\mathrm{k}}$ is the gain for the $\mathrm{k}^{\text {th }}$ trial, $(\Delta \mathrm{L})_{\mathrm{i}}$ defines the desired movement for that trial, $S_{\text {mean }}$ is the mean value for the input signal $\mathrm{S}$ in a given trial, and $\mu$, the adaptability constant, determines the rate of adaption.

Given a gain for trial k-1 and data from the trial, the gain for trial $\mathrm{k}$ will be a weighted average of gain from trial $\mathrm{k}-1$ and a gain that would have resulted in the least amount of error for trial k-1. The weights are determined by the adaptability constant $\mu$.

Using the LMS adaptive algorithm, the system can change its gain to match a change in the scale of the input signal in order to output a signal for positioning a linear actuator

\section{Control Signal Conditioning and Output}

For each cycle, a Labview (National Instruments; Austin, TX) program detects whether the control signal exceeded a threshold implemented to prevent movement due to noise:

$$
x_{i}=x_{i-1}+g s^{\prime}
$$

In equation (4), $g$ is the gain, $s$ ' is the thresholded control signal, and $x_{i}$ is the position the linear actuator is set to at the end of the $i$ th iteration.

The signal written to a UDP port by BCI2000 is read by Labview. This signal is the input signal to the classifier that determines how the linear actuator will act. The extension of the linear actuator is governed by

$$
\begin{aligned}
& x=L_{j-1}+G S \\
& L_{j}=\left\{\begin{array}{cl}
0 & x<0 \\
L_{\max } & x>L_{\text {max }} \\
x & \text { else }
\end{array}\right.
\end{aligned}
$$

where $L_{j}$ is the extension length of the linear actuator for iteration $\mathrm{j}, \mathrm{L}_{\max }$ is the maximum length of the linear actuator, $\mathrm{G}$ is the gain, and $\mathrm{S}$ is the input signal from BCI2000. The actuator position is then changed by writing to the actuator microcontroller via a USB port.

\section{E. Mechanical Orthosis}

The hand orthotic (Becker Oregon TAL100 Talon) was prefabricated to couple wrist motion to the opening and closing of the hand. This coupling was replaced with a linear actuator (Firgelli Miniature Linear Motion Series L16), shifting the control of the device to the Labview algorithm. 
The linear actuator operates according to pulse-width modulation (PWM) control signals received from Labview.



Figure 3 - prefabricated hand orthotic fitted with linear actuator for control of finger position

\section{RESULTS}

Our design was tested with four healthy subjects to verify the ability to use non-conventional signals from the contralesional cortex to control a hand on the same side of the body. We found that:

1. Hand movement correlates with EEG signals from the contralesional hemisphere.

2. Our design was successful in using these signals to coordinate a motor-driven orthosis.

Results of the screening procedure described above are shown in Figure 4 for a healthy subject. The correlation between the left hand movement condition and rest condition are evaluated using $R^{2}$ values per electrode channel (y axis) and per frequency bin (x axis). Channels names are shown according to the 10-20 EEG system. Bins with high $R^{2}$ values indicate significant power difference between movement and rest conditions and as such are good candidates for control signals. Clusters (dotted red circles) of high correlation are noted around the $12 \mathrm{~Hz}$ bins in $\mathrm{F} 3$ through P7 and also in channel F3 around the $22 \mathrm{~Hz}$ bin.

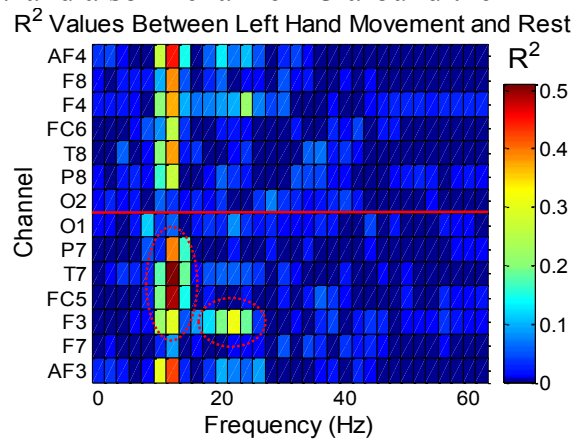

Figure $4-\mathbf{R}^{2}$ between left hand movemnet and rest across bins of electrode channel and frequency

Power of the F3 electrode during conditions of left hand movement and conditions of rest is shown in Figure 5. Movement produces even related desynchronization (ERD) in frequency bins around $12 \mathrm{~Hz}$ and $22 \mathrm{~Hz}$ as physiologically expected [3].

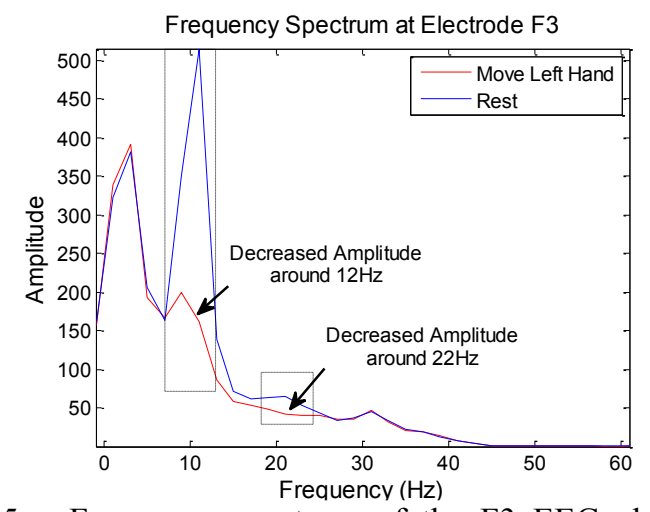

Figure 5 - Frequency spectrum of the F3 EEG electrode location during conditions of left hand movement (red curve) and conditions of rest (blue curve).

Closer examination of the $12 \mathrm{~Hz}$ bin shows distinct distributions of power during rest and left hand movement conditions (Figure 6). 2-tailed t-test confirms the difference between the two conditions is significant at better than the $\mathrm{p}=0.05$ level.

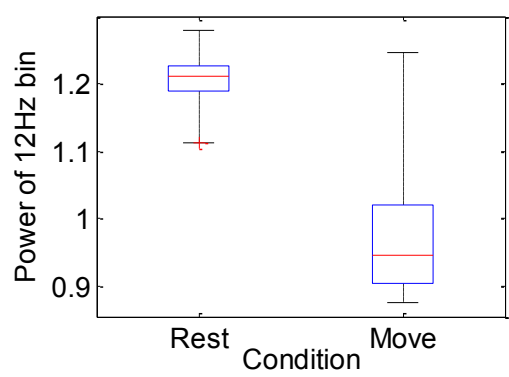

Figure 6 - Boxplot of $12 \mathrm{~Hz}$ bin power during left hand movement and rest conditions. Box describes $1^{\text {st }}$ through $3^{\text {rd }}$ quartiles and median. Whiskers describe range of data.

Figure 7 shows a topographical color map of $R^{2}$ correlation values between left hand movement and rest conditions for $12 \mathrm{~Hz}$ (left) and $22 \mathrm{~Hz}$ (right) brainwaves. Relatively high correlations are seen bilaterally in electrode F3 at $12 \mathrm{~Hz}$. The strength of this $12 \mathrm{~Hz}$ correlation allows for differentiation between left hand movement and rest conditions and was used as the control signal for all subjects. The $22 \mathrm{~Hz}$ band shows unilateral correlation and thus differentiates between left and right hand movement. While this experiment did not explore the use of the $22 \mathrm{~Hz}$ signals, they could provide an additional degree of freedom in future device control.
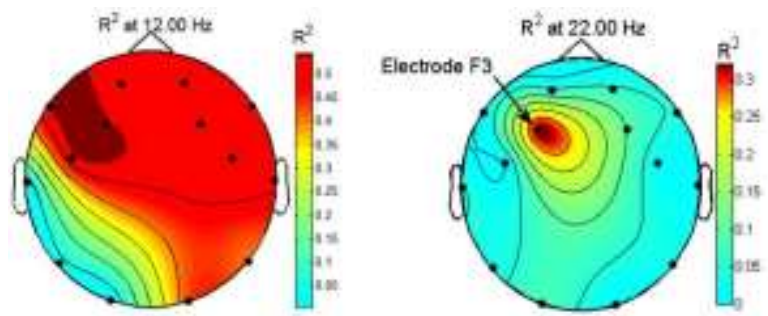

Figure 6-Topographical color maps of $\boldsymbol{R}^{\mathbf{2}}$ values for $12 \mathrm{~Hz}$ and $22 \mathrm{~Hz}$. Note the colormap scales are not the same. 
Using the power of the $12 \mathrm{~Hz}$ bin of channel $\mathrm{F} 3$, we generated control signals to move a cursor on a computer screen. The modulation of the control signal during imagined or actual hand movement gave the subject control of 1 dimensional cursor movement and orthosis position, and the subject was tasked with moving the cursor to a target that randomly appeared on either side of a computer screen. Through 10 sets of trials with non-impaired individuals we were able to achieve an $81.3 \%$ success rate for this task. Figure 7 presents a trace of target and actual orthosis positioning.

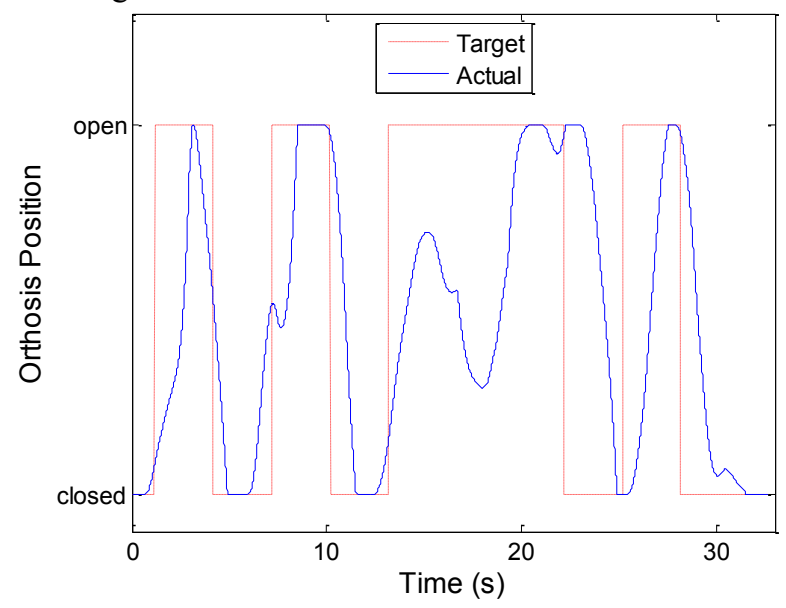

Figure 7 - Sample of orthosis positioning during a block of randomized trials of open and closed target positions. Actual position follows target position after a delay from windowing. Accuracy reflects correlation of $12 \mathrm{~Hz}$ signal.

Video verification of project success can be found at http://www.youtube.com/watch?v=4-7A5Q-kz-M

\section{DISCUSSION}

Combining the discovery of motor related signals in the undamaged brain hemisphere and consumer electronics, our device provides a novel method for rehabilitation for stroke survivors. We were able to process EEG signals for realtime hand control with accuracy and $\mathrm{R}^{2}$ values higher than previous studies [2]. Note that electrode locations do not cover typical motor cortex locations due to limitations of device design. Recent evidence suggests that combining BCIs and orthotic devices induces neural plasticity and improves motor function [1]. Furthermore, the potential for recovery is unhampered by the severity of neural pathway injury since we circumvent the entire injured pathway. The hope is that, with the help of BCIs, remaining contralesional areas of the brain can be trained to take over motor control of the impaired hand.

Synthesizing consumer electronics with the new technique of ipsilateral cortex recording has yet to be done. Compared to devices such as, Myomo of Neuro-robotic Systems ${ }^{\circledR}$, the Bioness H200 from Ness ${ }^{\circledR}$, and Hand Mentor from Kinetic Muscles Inc., our device facilitates plasticity most directly, is cheaper and more portable, and can be used even in cases of severe damage to neural pathways. Allowing patients to regain hand control with their thoughts will provide encouragement to continue with a therapy. Affordability and minimal need for therapist supervision, our device makes inhome treatment a very practical possibility.

Future development will be directed to expanding the system's ability to adapt to spatially non-stationary signals. Implementing adaptive spatial filters or an adaptive classifier that finds the strongest correlated channel automatically and continuously would improve robustness for a long-term outpatient orthosis. More importantly, spatial and temporal filters that remove artifacts from eye blinks, EMG, and breathing [8], are essential to the device performance outside of a research setting.

\section{ACKNOWLEDGMENT}

The undergraduate student authors would like to thank D. Bundy for providing technical support and comments on the manuscript and E. Leuthardt for advice throughout the work.

\section{REFERENCES}

[1] D Broetz and et al, "Combination of brain-computer interface training and goal-directed physical therpy in chronic stroke: A case report," J Neurorehab and Neural Repair, pp. 674-679, 2010.

[2] Jonathan R Wolpaw and Dennis J McFarland, "Control of a two-dimensional movement signal by a noninvasive brain-computer interface in humans," Proc. Natl. Acad. Sci, vol. 101, no. 51, pp. 17849-17854, December 2004.

[3] Kimberly J Wineski et al., "Unique cortical physiology associated with ipsilateral hand movements and neuroprosthetic implications," Stroke, pp. 3351-3359, 2009.

[4] Karunesh Ganguly et al., "Cortical representation of ipsilateral arm movement in monkey and man," The Journal of Neuroscience, vol. 29, no. 41, pp. 1294812956, October 2009.

[5] G Schalk, D McFarland, T Hinterberger, N Bribaumer, and J Wolpaw, "BCI2000: a general-purpose braincomputer interface (BCI) system," IEEE Transactions on Biomedical Engineering, pp. 1034-1043, 2004.

[6] Dennis J McFarland, Lynn M McCane, Stephen V David, and Jonathan R Wolpaw, "Spatial filter selection for EEG-based communication," Electroencephalography and Clinical Neurophysiology, vol. 103, pp. 386-394, 1997.

[7] Ernst Niedermeyer and Fernando Lopes da Silva, Electroencephalography: basic principles, clinical applications, and related fields.: Lippincott Williams \& Wilkins, 2004.

[8] Carrie A Joyce, Irina F Gorodnitsky, and Marta Kutas, "Automatic removal of eye movement and blink artifacts from EEG data using blind component separation," Psychophysiology, vol. 41, 2004. 